\title{
Preparation and biological characterization of the mixture of poly(lactic-co-glycolic acid)/chitosan/Ag nanoparticles for periodontal tissue engineering
}

This article was published in the following Dove Medical Press journal: International Journal of Nanomedicine

\author{
Yanxiang Xue ${ }^{1,2}$ \\ Xiaofang Hong ${ }^{3}$ \\ Jie $\mathrm{Gao}^{1,2}$ \\ Renze Shen ${ }^{3}$ \\ Zhanchao $\mathrm{Ye}^{3}$
}

'Department of Stomatology, The Liwan Hospital of The Third Affiliated Hospital of Guangzhou Medical University, Guangzhou 510000 , China; ${ }^{2}$ Department of Stomatology, Southern Medical University Guangzhou, Guangzhou 5105I5, China; ${ }^{3}$ Department of Stomatology, Zhongshan Hospital of Xiamen University, Medical College of Xiamen University, Xiamen University, Xiamen 361000, China
Correspondence: Yanxiang Xue Department of Stomatology, The Liwan Hospital of the Third Affiliated Hospital of Guangzhou Medical University, No. 63, Duobao Road, Liwan District, Guangzhou 510000, China

Tel +86I85 59229112

Email 873I59094@qq.com

Renze Shen

Department of Stomatology, Zhongshan Hospital of Xiamen University, Medical College of Xiamen University, Xiamen University, Xiamen Hubin Road 20I,

Xiamen 361000, China

Tel +86 I57 II 567015

Email8578III98@qq.com
Objective: This study aims to produce nanoparticles of chitosan (CS), poly(lactic-co-glycolic acid) (PLGA), and silver and investigate the optimal composite ratio of these three materials for periodontal tissue regeneration.

Methods: PLGA nanoparticles (nPLGA), CS nanoparticles (nCS), and silver nanoparticles (nAg) were prepared. The antibacterial properties of single nanoparticles and their effects on the proliferation and mineralization of periodontal membrane cells were investigated. Different ratios of nPLGA and nCS were combined, the proliferation and mineralization of periodontal membrane cells were investigated, and based on the results, the optimal ratio was determined. Finally, nPLGA and nCS in optimal ratio were combined with nAg, and the effects of the complex of these three materials on the proliferation and mineralization of periodontal membrane cells were investigated and tested in animals.

Results: The single nanoparticles were found to have no cytotoxicity and were able to promote cell mineralization. $\mathrm{nCS}$ and $\mathrm{nAg}$ in low concentrations showed antibacterial activity; however, nAg inhibited cell proliferation. The nPLGA and nCS complex in 3:7 ratio contributed to cell mineralization and had no cytotoxicity. nPLGA/nCS/nAg complex, which had the optimal proportion of the three materials, showed no cytotoxicity and contributed to cell mineralization. Conclusion: $\mathrm{nPLGA} / \mathrm{nCS} / \mathrm{nAg}$ complex had no cytotoxicity and contributed to cell mineralization. The 3:7 ratio of $\mathrm{nPLGA} / \mathrm{nCS}$ and $50 \mu \mathrm{g} / \mathrm{mL} \mathrm{nAg}$ were found as the optimal proportion of the three materials.

Keywords: nanoparticles, bone regeneration, periodontitis

\section{Introduction}

Periodontitis is a chronic disease that reduces the integrity of the periodontal system and causes damage to the periodontal tissues, eventually leading to tooth loss. ${ }^{1-3}$ Numerous studies have been done to find out solutions for periodontitis ranging from autogenous bone graft materials to guided tissue regeneration/guided bone regeneration (GTR/ GBR). Thus far, optimal materials for the regeneration of lost periodontal tissues have not been found or made. Autogenous bone cannot be easily obtained, and the allograft and xenograft materials may cause immunological rejection in recipients. Synthetic biodegradable materials have shown certain advantages, reducing the possibility of immune rejection, with abundant sources and at low cost. Thus, synthetic materials have always been a research hotspot.

Poly(lactic-co-glycolic acid) (PLGA) is one of the synthetic polymers approved by the US Food and Drug Administration (FDA) and one of the most widely applied materials in pharmaceutics and tissue engineering. It is characterized by excellent 
biocompatibility, tunable mechanical property, and controllable degradation rate and is easy to fabricate by varying the copolymer ratio of lactic acid to glycolic acid. ${ }^{4-6}$ Chitosan (CS), a natural cationic polymer, is the alkaline deacetylated product of chitin and is similar to glycosaminoglycan in extracellular structure. CS is broadly employed in biomedicine and industry by virtue of its excellent biological characteristics, such as biodegradability, biocompatibility, nontoxicity, relative hydrophilicity, and hemostatic and antibacterial activity. ${ }^{7-9}$ At present, there is no material that can completely satisfy the regeneration of periodontal tissue. Synthetic polymers have suitable mechanical and biological properties but lack biocompatibility, whereas natural polymers have excellent hydrophilicity and biocompatibility but lack the essential mechanical characteristics. Thus, by mixing different materials characterized by different properties, such as natural polymers with inorganic materials or synthetic polymers, numerous research groups have strived to design and fabricate periodontal materials for GTR/GBR with the requisite features and properties. ${ }^{10-12}$

Dental plaque biofilm is considered as the initial symptom of a periodontal disease. ${ }^{13}$ The key to successful treatment of a periodontal disease is the effective control of the dental plaque. Antibiotics are critical for the inhibition of the bacterial growth, but they also lead to emergence of drug resistance. Silver nanoparticles (nAg) are one of the most broadly applied antibacterial agents and exhibit broad-spectrum antibacterial activity. ${ }^{14}$ Besides the antibacterial property, nAg also have antifungal, anti-inflammatory, antiviral, and anticancer effect and lower drug resistance. Thus, $\mathrm{nAg}$ have been widely used in medical dressings, implants, dental materials, surgical masks, water filtration units, personal care items, etc. due to their unique characteristics. In this study, we added nAg to achieve long-term release of silver ions for promoting antibacterial resistance and to make the resistance remain longer.

Alveolar bone may undergo a slight reduction in the range of a few millimeters, but the effect of this reduction is highly significant. It is extremely difficult to introduce materials in such a small space, so nano-sized particles may be used for this purpose. Nanomaterials mimic the ultrastructure of defective tissues to the greatest extent and the nanostructure of extracellular matrix, increase the success rate of therapy, and offer promising applications. ${ }^{15}$ PLGA and CS can be easily combined as nanoparticles using various methods. PLGA nanoparticles (nPLGA) and CS nanoparticles (nCS) have been broadly used as drug delivery systems. ${ }^{16,17}$ The nano-sized materials are the first choice in any periodontal surgery, and hence, there is a need to prepare nanoparticles.
Thus far, no study has been performed on the mixture of nPLGA, nCS, and nAg. To our knowledge, this is the first study to prepare periodontal tissue regeneration materials by combining these three materials.

The aim of this study is to prepare a material that is harmless to cells, can improve the cell mineralization, and decrease the recurrence rate of periodontal disease. In this study, nanoparticles of PLGA, CS, and Ag were prepared, and the cytotoxicity of single components and their effect on cell mineralization were studied. After preliminary understanding of the properties of the materials, nPLGA and nCS were recombined, and the cytological properties were tested again. Finally, nAg were added to investigate the effect on cytotoxicity and mineralization, and the optimal composite ratio of the three materials was determined.

\section{Materials and methods}

PLGA (lactic acid:glycolic acid, 75:25), with a viscosity of $0.61 \mathrm{dL} / \mathrm{g}$, was purchased from Shandong Institute of Medical Instruments. CS powders with a degree of deacetylation of $95 \%$, with a viscosity of 40-100 MPa.s, were obtained from Tiengene Bio-Technique Co. Ltd (Guangzhou, China).nAg (partial size $20 \mathrm{~nm}$ ) powders were purchased from Shanghai Chaowei Nanotechnology Co. Ltd. DMEM (HyClone), FBS (Thermo Fisher Scientific), 0.25\% trypsin (Genom), collagenase I (Biosharp), PrimeScript ${ }^{\circledR}$ RT reagent kit (Takara), SYBR Premix DimerEraser kit (Takara), and other reagents used were all of analytical grade.

\section{nPLGA and nCS preparation nPLGA preparation}

Using a modified oil-in-water emulsion solvent evaporation method, nPLGA were prepared. ${ }^{1}$ For this, $100 \mathrm{mg}$ PLGA was dissolved in $10 \mathrm{~mL}$ acetone and slowly poured into $40 \mathrm{~mL}$ $2 \%$ polyvinyl alcohol. Then, the mixture was stirred on a magnetic stirrer at an ambient temperature. The mixture was stirred continuously at $800 \mathrm{rpm}$ for 8 hours, and the organic solvent was evaporated by stirring. The nanoparticles were obtained by centrifugation at $1,500 \mathrm{rpm}$ for 20 minutes, washed three times with deionized water, and dried naturally. The nPLGA were sterilized by $\gamma$-rays and stored before further application.

\section{nCS preparation}

nCS were prepared through interaction with sodium tripolyphosphate (TPP) polyanion using the ionic gelation method. ${ }^{2}$ For this, $40 \mathrm{mg}$ CS powder was dissolved in $40 \mathrm{~mL}$ of $1 \%$ glacial acetic acid by stirring. Then, $10 \mathrm{~mL}$ of $0.1 \%$ TPP solution was instilled into the CS solution dropwise for about 15 seconds and was stirred at 1,000 rpm using a 
magnetic stirrer. The nanoparticles formed spontaneously were collected through centrifugation at 15,000 rpm for 20 minutes. The supernatant was discarded, and nCS were rinsed with deionized water three times and then dried naturally. The nCS were sterilized by $\gamma$-rays and stored before further application.

\section{Characterization of the nanoparticles} Particle size, size distribution, and zeta potential

After the nanoparticles were formed, a $10 \mathrm{~mL}$ mixture was added into a glass bottle. The samples were diluted to a suitable density during measurement. The particles size, polydispersity index (PDI), and zeta potential of the samples were measured using a Malvern Zetasizer 3000HS. All measurements were performed three times.

\section{Morphology examination using transmission electron microscopy (TEM)}

A drop of nanoparticle suspension was instilled on a copper grid, the excess liquid was absorbed by the filter paper, and the grid was dried at an ambient temperature. The morphology of nanoparticles was then analyzed using TEM.

\section{Cytotoxicity and proliferation}

\section{Cell culture}

Primary human periodontal ligament cells (hPDLCs) were cultured, identified, and utilized in this study referring to the methods previously reported by our group. ${ }^{18,22}$

\section{Effect of nPLGA, nCS, and nAg on cytotoxicity, proliferation, and mineralization of PDLCs \\ Effect of nPLGA, nCS, and nAg on cytotoxicity and proliferation of PDLCs}

To prepare $1 \mathrm{mg} / \mathrm{mL}$ PLGA, 1 and $2 \mathrm{mg} / \mathrm{mL}$ nPLGA, $1 \mathrm{mg} / \mathrm{mL} \mathrm{CS}, 1$ and $2 \mathrm{mg} / \mathrm{mL} \mathrm{nCS}$, and $100 \mu \mathrm{g} / \mathrm{mL} \mathrm{nAg}$ suspensions, a suitable medium was added into a $4 \mathrm{~mL}$ tube containing PLGA, nPLGA, CS, nCS, and nAg, respectively. PDLCs were seeded into 96-well plates at a density of $3-5 \times 10^{3}$ cells/well. nPLGA group and nCS group were added with $100 \mu \mathrm{L}$ material suspension into the corresponding wells, and the final concentration of nAg group in the wells was adjusted to $2,10,20,30,40$, and $50 \mu \mathrm{g} / \mathrm{mL}$, respectively. The cytotoxicity and proliferation of PDLCs was evaluated using MTT assay. The cytotoxicity and proliferation of the cells were evaluated on days 1,2, and 3 and 1,2,3, 5, and 7, respectively. On the day of test, $20 \mu \mathrm{L}$ MTT solution at a concentration of $5 \mathrm{mg} / \mathrm{mL}$ was added to the wells and cells were cultured for 4 hours at $37^{\circ} \mathrm{C}$ in humid environment with $5 \% \mathrm{CO}_{2}$. After the culturing period, the solution was removed gently followed by the addition of $150 \mu \mathrm{L}$ dimethylsulfoxide. The plate was vibrated for 10 minutes before the measurement. The absorbance was read at $490 \mathrm{~nm}$ using a SpectraMax M5. Relative growth rate (RGR) was used as a measure of cytotoxicity (Table 1). The experiment was repeated three times.

$$
\mathrm{RGR}=\frac{\mathrm{B}-\mathrm{A}}{\mathrm{A}} \times 100 \%
$$

where A denotes the absorbance of negative control (NC) containing only cells and $\mathrm{B}$ is the absorbance of experimental group containing different materials.

\section{Mineralization assay}

\section{Alizarin Red S staining}

PDLCs were seeded into six-well plates at a density of $1-5 \times 10^{5}$ cells/well with $1.5 \mathrm{~mL}$ complete culture medium. Besides $\mathrm{nAg}, 1 \mathrm{~mL}$ of the abovementioned suspensions was added into six-well plates when the cell confluency was $80 \%$. After 3 days, hPDLCs were induced with a mineralized solution supplemented with $10 \mathrm{mmol} / \mathrm{L} \beta$-glycerol phosphate, $50 \mu \mathrm{L} / \mathrm{mL}$ ascorbic acid, and $10 \mathrm{~mol} / \mathrm{L}$ dexamethasone in complete medium for 3 weeks, respectively. The induced cells were washed three times with PBS and fixed in methanol for 10 minutes at an ambient temperature. The cells were washed three times with PBS and stained with $1 \%$ Alizarin Red S (ARS) for 10 minutes. After several washes with PBS, the cells were observed using an optical microscope.

\section{Real-time quantitative PCR (RT-qPCR) analysis of gene expression}

At day 21, the total RNA was isolated from hPDLCs using TRIzol in accordance with the manufacturer's instructions. An equivalent amount of RNA samples was reversetranscribed for the first-strand cDNA synthesis using the PrimeScript ${ }^{\circledR}$ RT reagent kit. RT-qPCR was performed on a LightCycler 480 using SYBR Premix DimerEraser

Table I Evaluation criteria of cytotoxicity

\begin{tabular}{l|l}
\hline RGR & Toxicity grading \\
\hline 100 & 0 \\
$75-99$ & 1 \\
$50-74$ & 2 \\
$25-49$ & 3 \\
$1-24$ & 4 \\
0 & 5 \\
\hline
\end{tabular}

Abbreviation: RGR, relative growth rate. 
kit. The relative expression of the genes was normalized against the housekeeping gene $\beta$-actin. All samples were assayed three times, and the experiment was performed three times. The cycle threshold $(\mathrm{Ct})$ value of each target gene was normalized against the $\mathrm{Ct}$ value of $\beta$-actin. The relative expression of the target gene was calculated using $2^{-\Delta \Delta \mathrm{Ct}}$ method. The primer sequences of OCN, ALP, OPN, and $\beta$-actin are shown in Table 2.

\section{Antibacterial studies}

To prepare 1 and $5 \mathrm{mg} / \mathrm{mL}$ of CS and $\mathrm{nCS}, 1 \mathrm{mg} / \mathrm{mL}$ of $\mathrm{nAg}$, and $5,10,15$, and $20 \mathrm{mg} / \mathrm{mL}$ of PLGA and nPLGA suspensions, respectively, a certain amount of Luria broth was added into $2 \mathrm{~mL}$ Eppendorf tubes containing different materials. After the test, $\mathrm{CS}$ and $\mathrm{nCS}$ suspensions were diluted to final concentrations $(800 \mu \mathrm{g} / \mathrm{mL}$ and 1 , 2 , and $5 \mathrm{mg} / \mathrm{mL}$ ). $\mathrm{nAg}$ suspensions were diluted to the concentrations of 400,600 , and $800 \mu \mathrm{g} / \mathrm{mL}$ and $1 \mathrm{mg} / \mathrm{mL}$, respectively. Gram-negative bacterium Escherichia coli was employed to check the antibacterial properties of the materials, and the effectiveness was evaluated using plate colony-counting methods. For this, $1 \mu \mathrm{L} E$. coli suspension was instilled into $50 \mu \mathrm{L}$ material suspensions at different concentrations, which was shook for 12 hours at a constant temperature in an incubator shaker. The suspensions were appropriately diluted, and $1 \mu \mathrm{L}$ bacterial solution was transferred to agar plate and cultured for 24 hours at $37^{\circ} \mathrm{C}$ to calculate the colonies. Every concentration was tested on three plates, and this experiment was repeated five times.

nPLGA was mixed with the same amount of nCS and nAg to evaluate the influence of nPLGA on the antibacterial effect of $\mathrm{nCS}$ and $\mathrm{nAg}$. The concentration was set at $800 \mu \mathrm{g} / \mathrm{mL}$. The experiment was performed on negative control, $\mathrm{nCS}$, nAg, nAg/nCS, nAg/nPLGA, nCS/nPLGA, and nAg/nCS/ nPLGA groups. Other procedures were the same as those mentioned above.

Table 2 Primer sequences

\begin{tabular}{|c|c|c|}
\hline \multirow{2}{*}{$\begin{array}{l}\text { Gene } \\
\beta \text {-Actin }\end{array}$} & \multicolumn{2}{|c|}{ Primer sequences } \\
\hline & Forward primer & GCGCGGCTACAGCTTCA \\
\hline & Reverse primer & СТССTTAATGTCACGCACGAT \\
\hline \multirow[t]{2}{*}{$\mathrm{OCN}$} & Forward primer & GGCAGCGAGGTAGTGAAGA \\
\hline & Reverse primer & CCTGAAAGCCGATGTGGT \\
\hline \multirow[t]{2}{*}{ ALP } & Forward primer & CCAAAGGCTTCTTCTTGCTG \\
\hline & Reverse primer & CCACCAAATGTGAAGACGTG \\
\hline \multirow[t]{2}{*}{ OPN } & Forward primer & TGAAACGAGTCAGCTGGATG \\
\hline & Reverse primer & TGAAATTCATGGCTGTGGAA \\
\hline
\end{tabular}

Effect of nPLGA/nCS mixture on cytotoxicity, proliferation, and mineralization of PDLCs

Effect of nPLGA/nCS mixture on cytotoxicity and proliferation of hPDLCs

As mentioned above, a suitable concentration of each material was selected to perform this study. nPLGA and nCS were added together to a 96-well plate at the ratios of $9: 1,8: 2$, and 7:3. MTT assay was employed to evaluate the cytotoxicity and proliferation of PDLCs. Other procedures were the same as those mentioned above.

\section{Effect of $n P L G A / n C S$ mixture on mineralization of hPDLCs}

\section{ARS staining}

One milliliter of nPLGA and nCS were added together to a six-well plate at the ratios of 9:1, 8:2, and 7:3. hPDLCs were induced with the mineralized solution for 21 days. The well without materials was used as negative control. Three wells were used for each group. After incubation, the wells were stained by ARS, and the developed mineralized nodule was observed using an optical microscope.

\section{RT-qPCR analysis}

To measure the mRNA expression of OCN, ALP, and OPN, the effect of the mixture on the osteogenic differentiation of hPDLCs was assessed using RT-qPCR. The total RNA was extracted using TRIzol reagent. cDNA was synthesized from $500 \mu \mathrm{g}$ of total RNA following the manufacturer's protocol. Other procedures were the same as those mentioned above.

\section{Effect of $\mathrm{nPLGA} / \mathrm{nCS}$ mixture on cytotoxicity, proliferation, and mineralization of PDLCs Effect of $n P L G A / n C S / n A g$ mixture on hPDLCs}

The optimal ratio of nPLGA and nCS and the suitable concentration of $n \mathrm{Ag}$ were selected according to the results of above assays. First, $100 \mu \mathrm{L}$ of the mixture of nPLGA and nCS was added. Then, a suitable volume of $\mathrm{nAg}$ was added to reach the selected concentration. After 1, 2, 3, 5, and 7 days of the addition of the material suspensions to the cells, MTT assay was performed. After the cells were cultured for 21 days with the mineral solution, they were stained with ARS, and the total RNA was extracted. Other procedures were the same as those mentioned above.

\section{Animal experiment Implantation of graft materials}

The animal experiment was conducted according to the ARRIVE guidelines and guidelines of the State Committee of 
Science and Technology of the People's Republic of China. Three New Zealand White rabbits (4-6 months old, weight 2-3 kg) were used for the animal experiment. Each rabbit was placed in a supine position with its limbs fixed on the operating table. To anesthetize the animal, Su Mian Xin II and $3 \%$ sodium pentobarbital solution ( $1 \mathrm{~mL} / \mathrm{kg}$ body mass) were administered intramuscularly. After the anesthetic had taken effect, the fur of the animal was shaved, and the skin was sterilized. An incision of a length of nearly $2.0 \mathrm{~cm}$ was created along the lower edge of the lower jaw to expose the lower edge of the mandible. The skin was cut, and the subcutaneous tissue was directly separated from the superficial fascia of this region to expose the lower edge of the mandible. In the molar area of the mandibular body, three $5 \times 3 \mathrm{~mm}$ cylindrical bone defects were created with a high-speed dental lathe. To decrease the heat generation and protect the bone tissue, the operating site was flushed with the isotonic saline during the operation. nPLGA/nCS/nAg and PLGA/CS/nAg graft materials were added to the defects from anterior to posterior as follows: PLGA/CS/nAg group, $\mathrm{nPLGA} / \mathrm{nCS} / \mathrm{nAg}$ group, and negative control. The periosteum was covered. Besides, the wound site and the layers of the incision were tightly sutured. The other side of the mandible was processed in the same way.

\section{Gross morphology and X-ray assay}

By injecting air into the ear veins, the rabbits were sacrificed at 2, 4, and 8 weeks after the operation. The soft tissues surrounding the mandible were stripped to fully obtain the mandible. The mandible was washed by flowing water to eliminate the blood and the residual soft tissues for gross examination and X-ray irradiation. Each mandibular specimen was irradiated and scanned using a dental X-ray machine placed on an X-ray film. The radio density in the defect areas of the mandible was observed and compared with the normal areas.

\section{Histological and histomorphological analysis}

The mandibular specimens were trimmed and placed in $4 \%$ paraformaldehyde for 24 hours. The specimens were decalcified in $17 \%$ EDTA solution for $6-8$ weeks in a $37^{\circ} \mathrm{C}$ thermostat water bath, dehydrated with a series of alcohol solutions $(70 \%, 80 \%, 90 \%, 95 \%, 100 \%)$, treated with xylene for transparency, and embedded in paraffin and sectioned to $4 \mu \mathrm{m}$ thickness. The conventional H\&E staining and histomorphological observation were performed on sections using an optical microscope. The presence and number of inflammatory cells including neutrophils and lymphocytes were observed under an optical microscope.

\section{Statistical analyses}

All the quantitative data were expressed as mean \pm SD. The statistical analysis was performed using one-way ANOVA. All statistical tests were analyzed with SPSS 20.0. A value of $P<0.05$ was considered statistically significant in this study.

\section{Results}

\section{Characterization of nPLGA and $n C S$}

nPLGA were successfully fabricated using an oil-in-water emulsion solvent evaporation method. The particle size and morphology of nanoparticles were measured and observed, respectively, using Malvern Zetasizer and TEM. The mean diameter of nanoparticles was estimated as $112.4 \pm 8.33 \mathrm{~nm}$, and the PDI was $0.13 \pm 0.05$ (Figure 1A). The surface charge is a critical parameter that reflects the physicochemical and biological stability of nanoparticles. Zeta potential values of the nPLGA were found to be $-25.4 \pm 2.6 \mathrm{mV}$ (Figure 1C). When the absolute zeta potential value was greater than $25 \mathrm{mV}$, nanoparticles were considered relatively stable. As revealed by TEM, nPLGA were spherical and regular in shape (Figure 1B).

nCS were prepared by ionic gelation with TPP at a mass ratio of $4: 1$. The size distribution and morphology of the nanoparticles are shown in Figure 2. The mean diameter and PDI of nCS were $180.3 \pm 11.2 \mathrm{~nm}$ and $0.37 \pm 0.07 \mathrm{~nm}$, respectively, as measured using the Malvern Zetasizer (Figure 2A). Zeta potential value of nCS was found to be $+34.0 \pm 0.8 \mathrm{mV}$ (Figure 2C). The nCS is spherical with clear structure (Figure 2B). The particle size measured from the TEM images was not consistent with that measured using the Malvern Zetasizer. The diameter of nanoparticles measured using TEM was smaller than that measured using Malvern Zetasizer. This was probably because the nanoparticles were gathered together when measured by Malvern Zetasizer and were surrounded by a water layer.

\section{Effect of nPLGA, nCS, and nAg on cytotoxicity and proliferation of hPDLCs}

Toxicity grading of nPLGA, nCS, and nAg for hPDLCs is listed in Tables 3-5, respectively. A material with a toxicity grade of 0 or 1 can be considered nontoxic according to ISO 10993-5. As shown in Tables 3 and 4, nPLGA or nCS had similar cytocompatibility as PLGA and CS. With the increase of concentration, nanoparticles maintained their cytocompatibility. nAg also maintained good cytocompatibility with the increase in concentration and time.

The results suggested that the cell numbers increased with the extension of period of culture with the three 

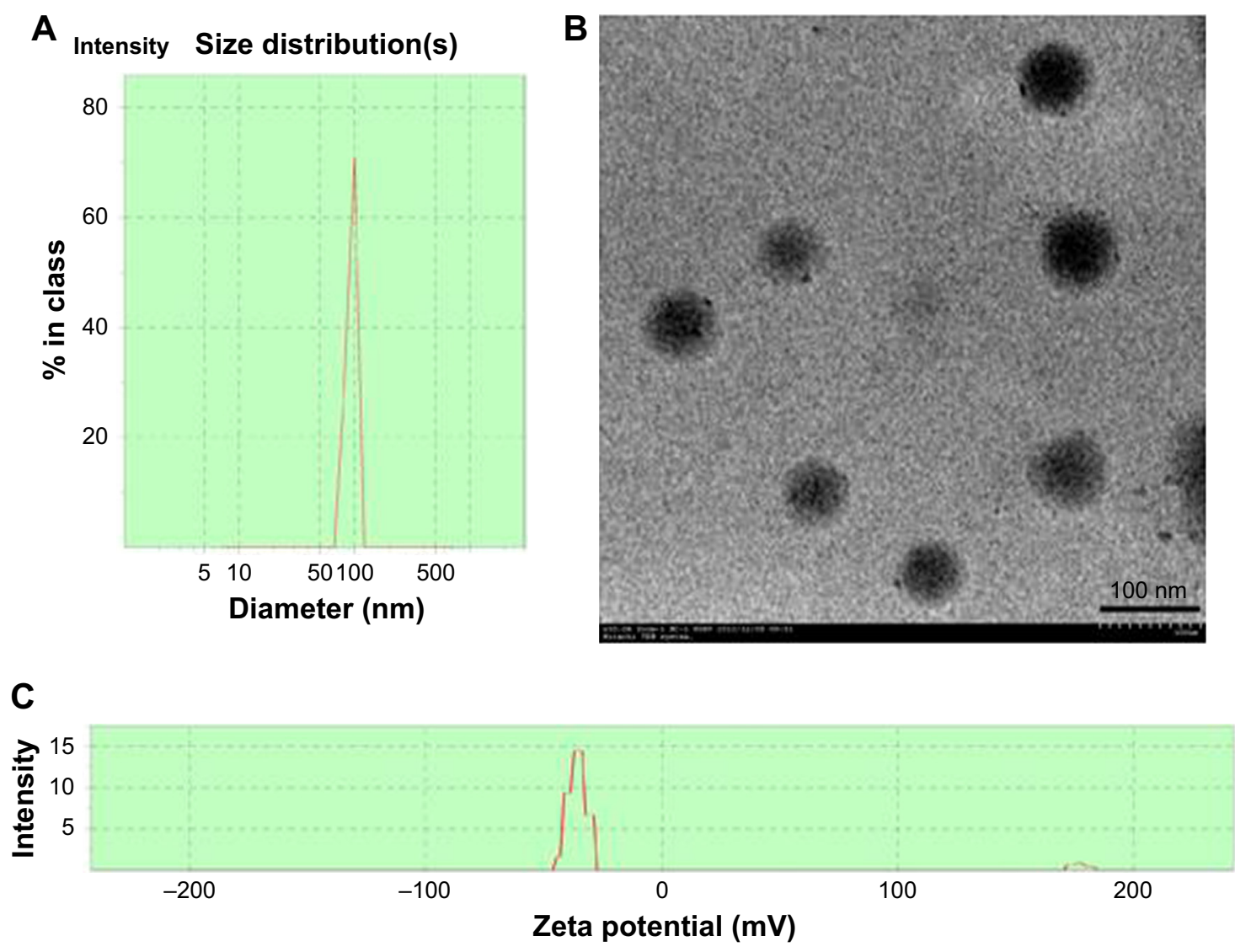

Figure I The characterization of nPLGA.

Notes: Malvern Zetasizer 3000HS size measurement of nPLGA (A). TEM images of nPLGA (B). Zeta potential of nPLGA (C).

Abbreviations: nPLGA, poly(lactic-co-glycolic acid) nanoparticles; TEM, transmission electron microscopy.

nanoparticles, as shown in Figure 3. The proliferation of cells was not affected by the structure and concentration of materials $(P>0.05)$, as shown in Figure $3 \mathrm{~A}$ and $\mathrm{B}$. The proliferation of cells in $2 \mathrm{nAg}, 10 \mathrm{nAg}, 20 \mathrm{nAg}$, and $30 \mathrm{nAg}$ groups did not differ from that of negative control group during the measurement. The proliferation of cells in 40 $\mathrm{nAg}$ and $50 \mathrm{nAg}$ groups was consistent and did not differ from that of the negative control group on the first 3 days, while the proliferation of cells was much slower than that of the control group on the fifth and seventh day, as shown in Figure 3C. Statistically, the proliferation rate of the two groups was significantly different from the control group $(P<0.05)$.

Table 3 Toxicity grading of nPLGA for hPDLCs

\begin{tabular}{l|l|l|l|l}
\hline & PLGA & 0.5 nPLGA & I nPLGA & 2 nPLGA \\
\hline I day & $98.7 \pm 2.2(\mathrm{I})$ & $87.4 \pm 1.6(\mathrm{I})$ & $99.4 \pm 7.7(\mathrm{I})$ & $103.2 \pm 4.8(0)$ \\
2 days & $94.1 \pm 8.0(\mathrm{I})$ & $96.1 \pm 6.7(\mathrm{I})$ & $95.7 \pm 8.6(\mathrm{I})$ & $99.1 \pm 6.2(\mathrm{I})$ \\
3 days & $105.6 \pm 6.0(0)$ & $102.9 \pm 6.0(0)$ & $97.8 \pm 9.2(\mathrm{I})$ & $107.5 \pm 7.4(0)$ \\
\hline
\end{tabular}

Note: The number stands for the corresponding concentration.

Abbreviations: hPDLCs, human periodontal ligament cells; nPLGA, PLGA nanoparticles; PLGA, poly(lactic-co-glycolic acid).

\section{Effect of $n P L G A, n C S$, and $n A g$ on mineralization of hPDLCs \\ ARS staining of hPDLCs}

More mineralized nodules were observed on the wells with materials than that on negative control group, as shown in Figure 4. The number and structure of nodules showed no significant difference with respect to the concentration of the same material.

\section{RT-qPCR analysis of gene expression in hPDLCs}

The expression of mineralized genes in both the nonnanomaterial group and the nanoparticle group was higher than that in the blank group, as shown in Figure 5. The ALP

Table 4 Toxicity grading of nCS for hPDLCs

\begin{tabular}{l|l|l|l|l}
\hline & $\mathbf{C S}$ & $\mathbf{0 . 5} \mathbf{n C S}$ & $\mathbf{I} \mathbf{n C S}$ & $\mathbf{2} \mathbf{n C S}$ \\
\hline $\mathrm{I}$ day & $98.5 \pm 2.5(\mathrm{I})$ & $100.3 \pm 3.4(0)$ & $97.6 \pm I .7(\mathrm{I})$ & $98.8 \pm 0.9(\mathrm{I})$ \\
2 days & $87.6 \pm \mathrm{I} .7(\mathrm{I})$ & $92.9 \pm 6.2(\mathrm{I})$ & $88.4 \pm 4.7(\mathrm{I})$ & $93.8 \pm 2.4(\mathrm{I})$ \\
3 days & $90.5 \pm 8.6(\mathrm{I})$ & $99.3 \pm 4.8(\mathrm{I})$ & $\mathbf{I} 02.6 \pm \mathrm{I} 2.3(0)$ & $98 . I \pm 7.3(\mathrm{I})$ \\
\hline
\end{tabular}

Note: The number stands for the corresponding concentration.

Abbreviations: CS, chitosan; hPDLCs, human periodontal ligament cells; nCS, CS nanoparticles. 

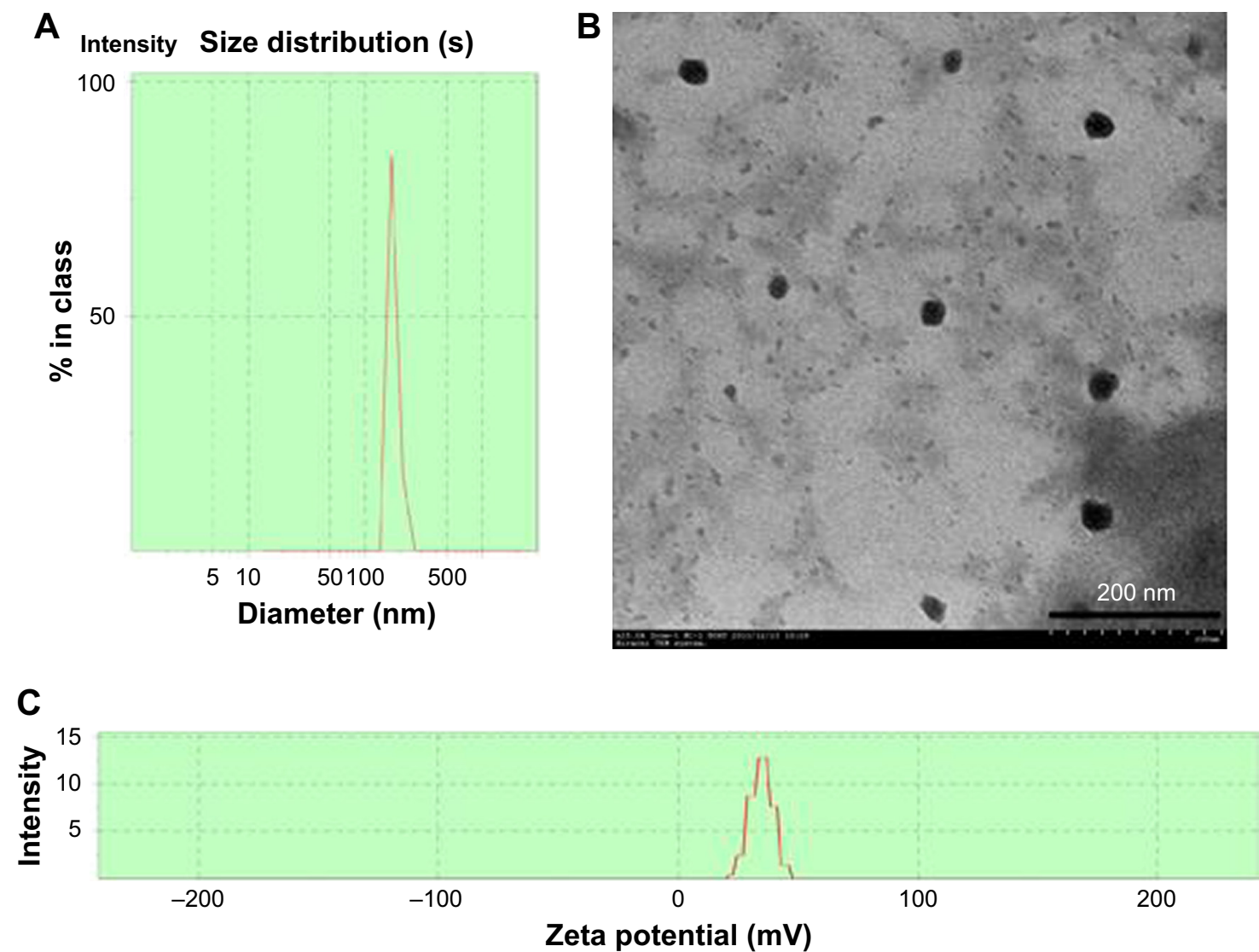

Figure 2 The characterization of nCS.

Notes: Malvern Zetasizer 3000HS size measurement of nCS (A). TEM images of nCS (B). Zeta potential of $n C S(C)$.

Abbreviations: $\mathrm{nCS}$, chitosan nanoparticles; TEM, transmission electron microscopy.

expression of the nanoparticle group was higher than that of the non-nanomaterial group.

\section{Antibacterial studies}

$\mathrm{nAg}$ at a concentration of $400 \mu \mathrm{g}$ showed strong bacteriostatic properties, and the antimicrobial activity rate was above $50 \%$. nCS were more bacteriostatic than CS. PLGA had no impact on the bacteriostatic properties of $\mathrm{nAg}$ and CS, as shown in Figure 6.

\section{Effect of nPLGA/nCS mixture on cytotoxicity and proliferation of hPDLCs}

No significant decrease in OD value was observed between the experimental group and the control group in the cell proliferation assay. Different proportions of $\mathrm{nPLGA} / \mathrm{nCS}$ showed no toxicity on cells and no obvious impact on their proliferation, as shown in Table 6 and Figure 7.

\section{Effect of $n P L G A / n C S$ mixture on the mineralization of hPDLCs \\ ARS staining of hPDLCs}

With low CS content, the number of cell mineralized nodules formed was less, but there was insignificant difference from the blank group (Figure 8, 9:1 and NC). High CS content improved the formation of cell mineralized nodules (Figure 8). This result was consistent with the results of mineralized gene detection (Figure 9).

Table 5 Toxicity grading of $\mathrm{nAg}$ for hPDLCs

\begin{tabular}{l|l|l|l|l|l|l}
\hline & $\mathbf{2} \mathbf{n A g}$ & $\mathbf{1 0} \mathbf{n A g}$ & $\mathbf{2 0} \mathbf{n A g}$ & $\mathbf{3 0} \mathbf{n A g}$ & $\mathbf{4 0} \mathbf{n A g}$ & $\mathbf{5 0} \mathbf{n A g}$ \\
\hline $\mathrm{I}$ day & $91.6 \pm 5.9(\mathrm{I})$ & $89.4 \pm \mathrm{II} .3(\mathrm{I})$ & $84.1 \pm 3.0(\mathrm{I})$ & $83.0 \pm 4.0(\mathrm{I})$ & $82.2 \pm 4 . \mathrm{I}(\mathrm{I})$ & $82.2 \pm 6.3(\mathrm{I})$ \\
2 days & $93.6 \pm 12.3(\mathrm{I})$ & $98.7 \pm 8.8(\mathrm{I})$ & $96.0 \pm 7.6(\mathrm{I})$ & $98.2 \pm I .5(\mathrm{I})$ & $91.9 \pm 4.8(\mathrm{I})$ & $93.1 \pm 5.5(\mathrm{I})$ \\
3 days & $98.2 \pm 4.0(\mathrm{I})$ & $98.4 \pm 4.2(\mathrm{I})$ & $97.6 \pm 6.4(\mathrm{I})$ & $94 . I \pm 6.1(\mathrm{I})$ & $96.7 \pm 2.0(\mathrm{I})$ & $92.7 \pm 2.8(\mathrm{I})$ \\
\hline
\end{tabular}

Note: The number stands for the corresponding concentration.

Abbreviations: hPDLCs, human periodontal ligament cells; $\mathrm{nAg}, \mathrm{Ag}$ nanoparticles. 
A

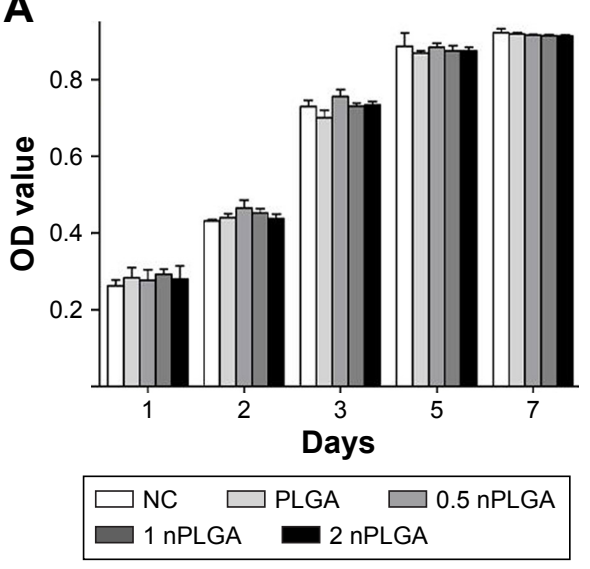

B

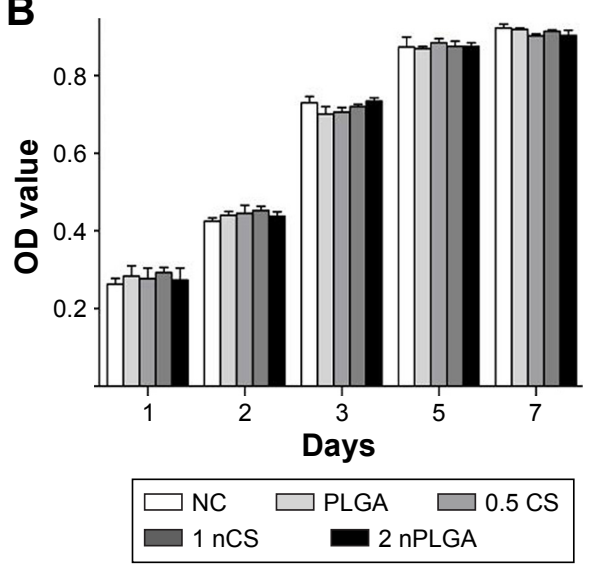

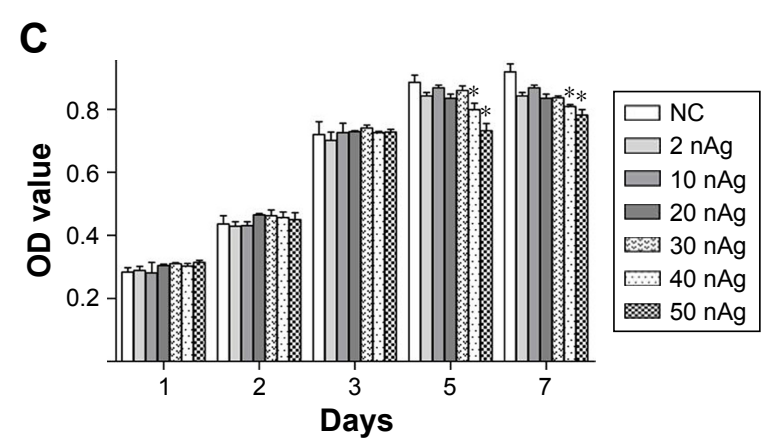

Figure 3 Effect of nPLGA, nCS, and nAg on the proliferation of hPDLCs.

Notes: Effect of $n P L G A(\mathbf{A})$. Effect of $n C S(B)$. Effect of $n A g(C)$. The number stands for the corresponding concentration. $* P<0.05$.

Abbreviations: CS, chitosan; hPDLCs, human periodontal ligament cells; nAg, silver nanoparticles; nCS, CS nanoparticles; nPLGA, PLGA nanoparticles; PLGA, poly(lacticco-glycolic acid).

\section{RT-qPCR analysis}

Effect of PLGA/CS/nAg mixture on cytotoxicity and proliferation of hPDLCs

No significant decrease in OD value was observed between the experimental group and the control group in the cell proliferation experiments. Different proportions of nPLGA/ $\mathrm{nCS} / \mathrm{nAg}$ showed no toxicity on cells, and no obvious effect on their proliferation (Table 7 and Figure 10).

\section{Effect of $n P L G A / n C S$ mixture on mineralization of hPDLCs}

ARS staining and RT-qPCR analysis of gene expression in hPDLCs

The cell mineralized nodules in the nanomaterials group and the non-nanomaterials group were significantly improved in

Table 6 Toxicity grading of $n P L G A / n C S$ in different ratios for hPDLCs

\begin{tabular}{l|l|l|l}
\hline & $9: 1$ & $8: 2$ & $7: 3$ \\
\hline I day & $104.8 \pm 3.3(0)$ & $100.4 \pm 3.5(0)$ & $101.4 \pm 2.1(0)$ \\
2 days & $97.2 \pm 0.9(1)$ & $102.3 \pm 4.8(0)$ & $101.0 \pm 4.1(0)$ \\
3 days & $102.8 \pm 2.8(0)$ & $96.9 \pm 0.6(1)$ & $99.6 \pm 1.2(1)$ \\
\hline
\end{tabular}

Abbreviations: $\mathrm{hPDLCs}$, human periodontal ligament cells; $\mathrm{nCS}$, chitosan nanoparticles; nPLGA, poly (lactic-co-glycolic acid) nanoparticles. comparison with NC group (Figure 11). The expression of mineralized genes such as OCN and OPN in the experimental group was higher than that in the NC group (Figure 12).

\section{Gross morphology and X-ray assay}

It can be observed from Figure 13 that bone defects recovered more rapidly after addition of implant materials. The bone recovery rate of $\mathrm{nPLGA} / \mathrm{nCS}$ group was greater than that of $\mathrm{nPLGA} / \mathrm{nCS} / \mathrm{nAg}$ group, while the recovery rate of both groups was greater than that of the blank group. The bone tissue recovery in the experimental group was better than that in the control group, and bone mineral density of the experimental group was higher than that of the control group.

Table 7 Toxicity grading of $n P L G A / n C S / n A g$ and PLGA/CS/nAg for hPDLCs $(P<0.01)$

\begin{tabular}{l|l|l}
\hline & $\mathbf{n P L G A} / \mathbf{n C S / n A g}$ & PLGA/CS/nAg \\
\hline $\mathrm{I}$ day & $94.2 \pm 3.6(\mathrm{I})$ & $96.4 \pm 2.8(\mathrm{I})$ \\
2 days & $97.3 \pm 1.7(\mathrm{I})$ & $98.5 \pm 4.0(\mathrm{I})$ \\
3 days & $95.0 \pm 0.8(\mathrm{I})$ & $97.6 \pm 3.3(\mathrm{I})$ \\
\hline
\end{tabular}

Abbreviations: hPDLCs, human periodontal ligament cells; $\mathrm{nAg}$, silver nanoparticles; nCS, chitosan nanoparticles; nPLGA, poly(lactic-co-glycolic acid) nanoparticles. 
A

NC

PLGA

1 nPLGA

2 nPLGA
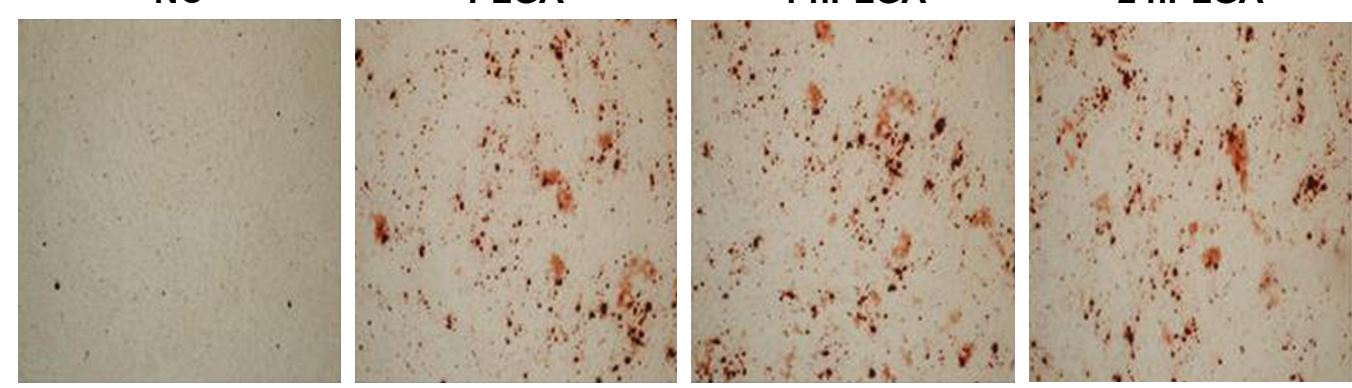

B

NC

CS

$1 \mathrm{nCs}$

2 nCS
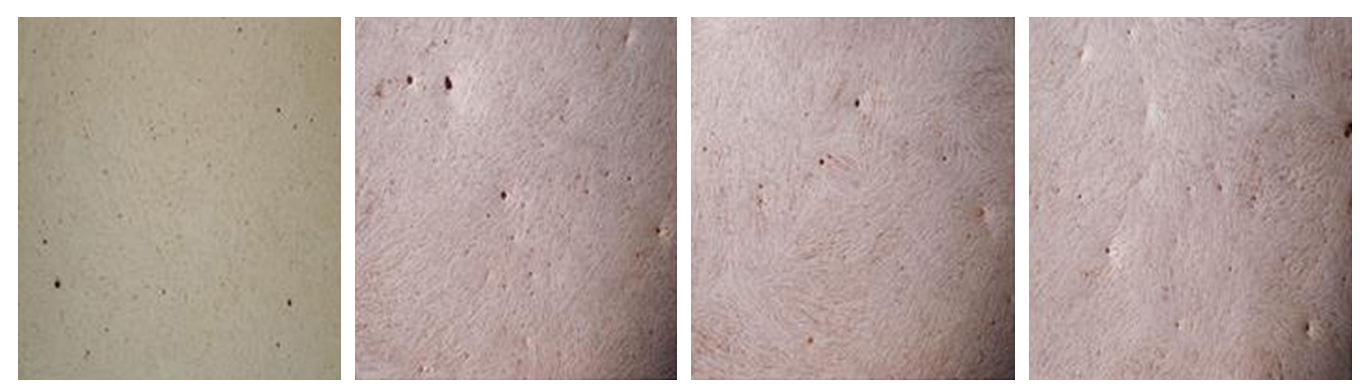

Figure 4 Alizarin Red S staining $(\times 100)$.

Notes: nPLGA groups (A). nCS groups (B).

Abbreviations: CS, chitosan; nCS, CS nanoparticles; nPLGA, PLGA nanoparticles; PLGA, poly(lactic-co-glycolic acid).
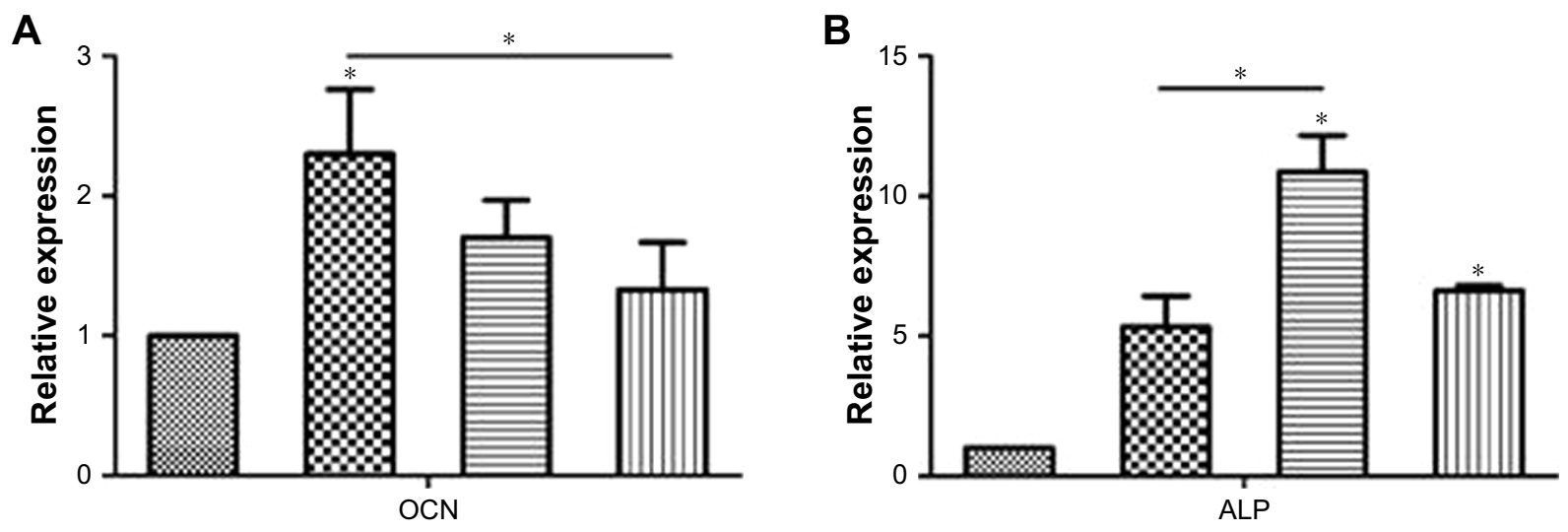

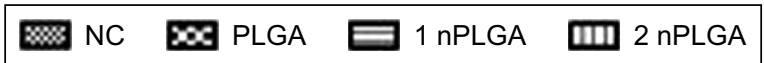
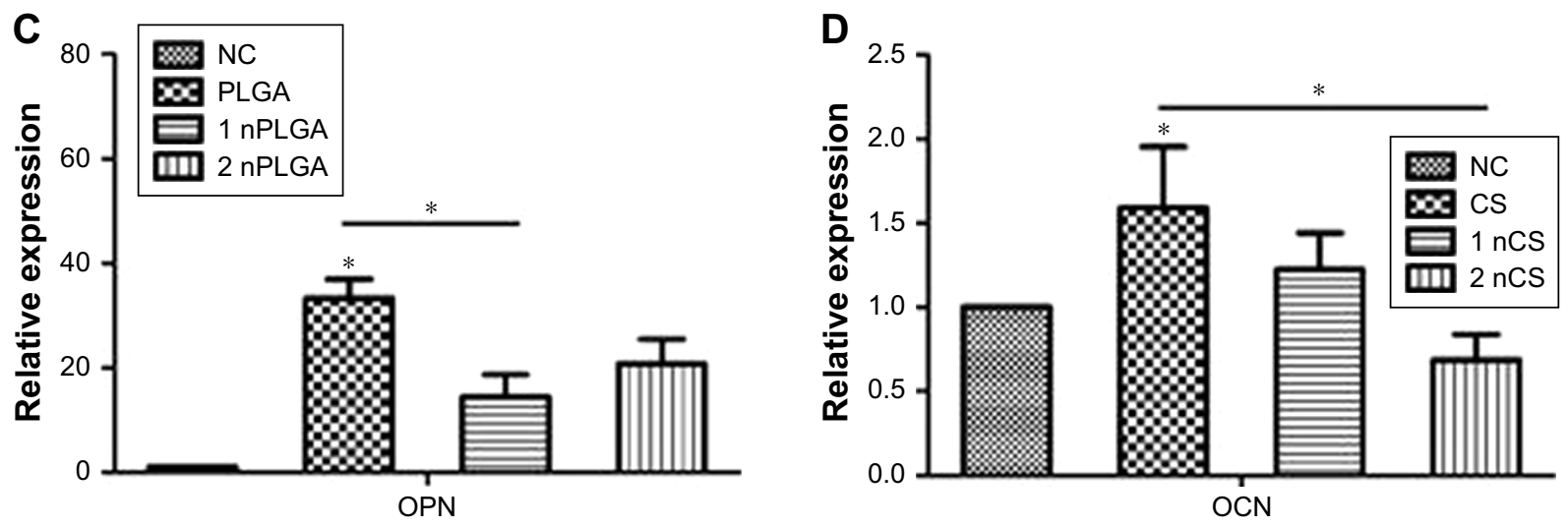

Figure 5 (Continued) 

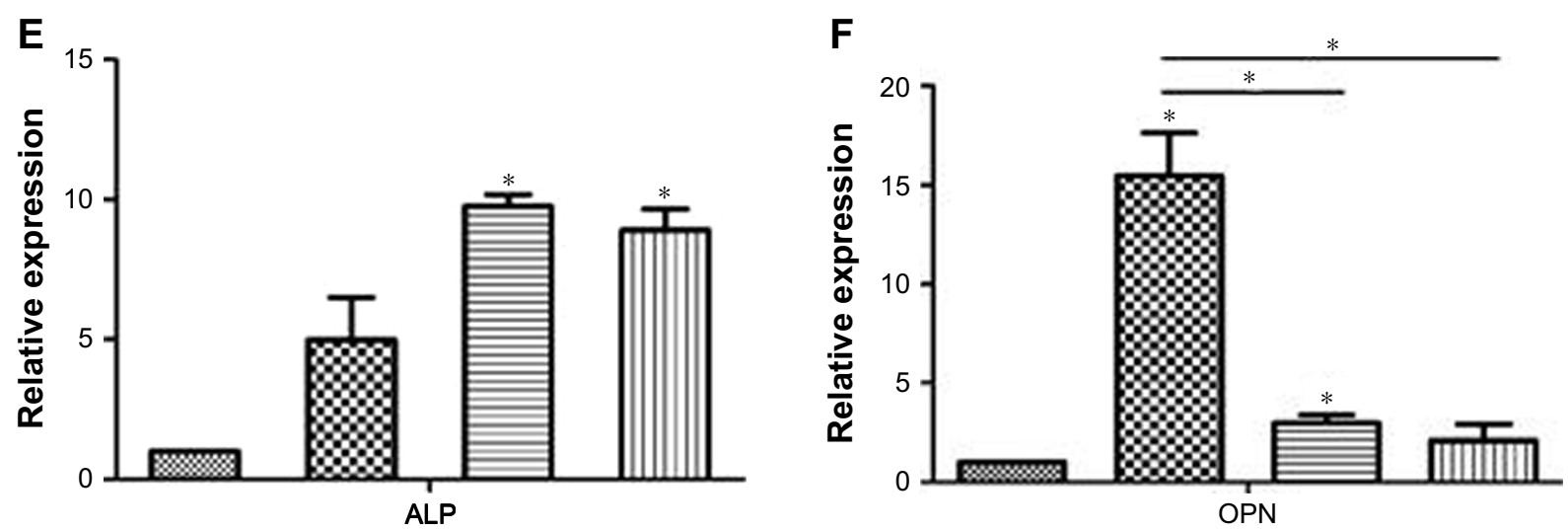

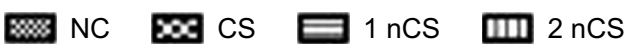

Figure 5 Real-time quantitative PCR analysis of the relative expression of osteogenic genes OCN, ALP, and OPN in hPDLCs cultured with different materials in osteogenic medium.

Notes: The relative mRNA expression of cells cultured with nPLGA groups (A-C) and cells cultured with $n C S(D-F) . * P<0.05$.

Abbreviations: CS, chitosan; hPDLCs, human periodontal ligament cells; nCS, CS nanoparticles; nPLGA, PLGA nanoparticles; PLGA, poly(lactic-co-glycolic acid).
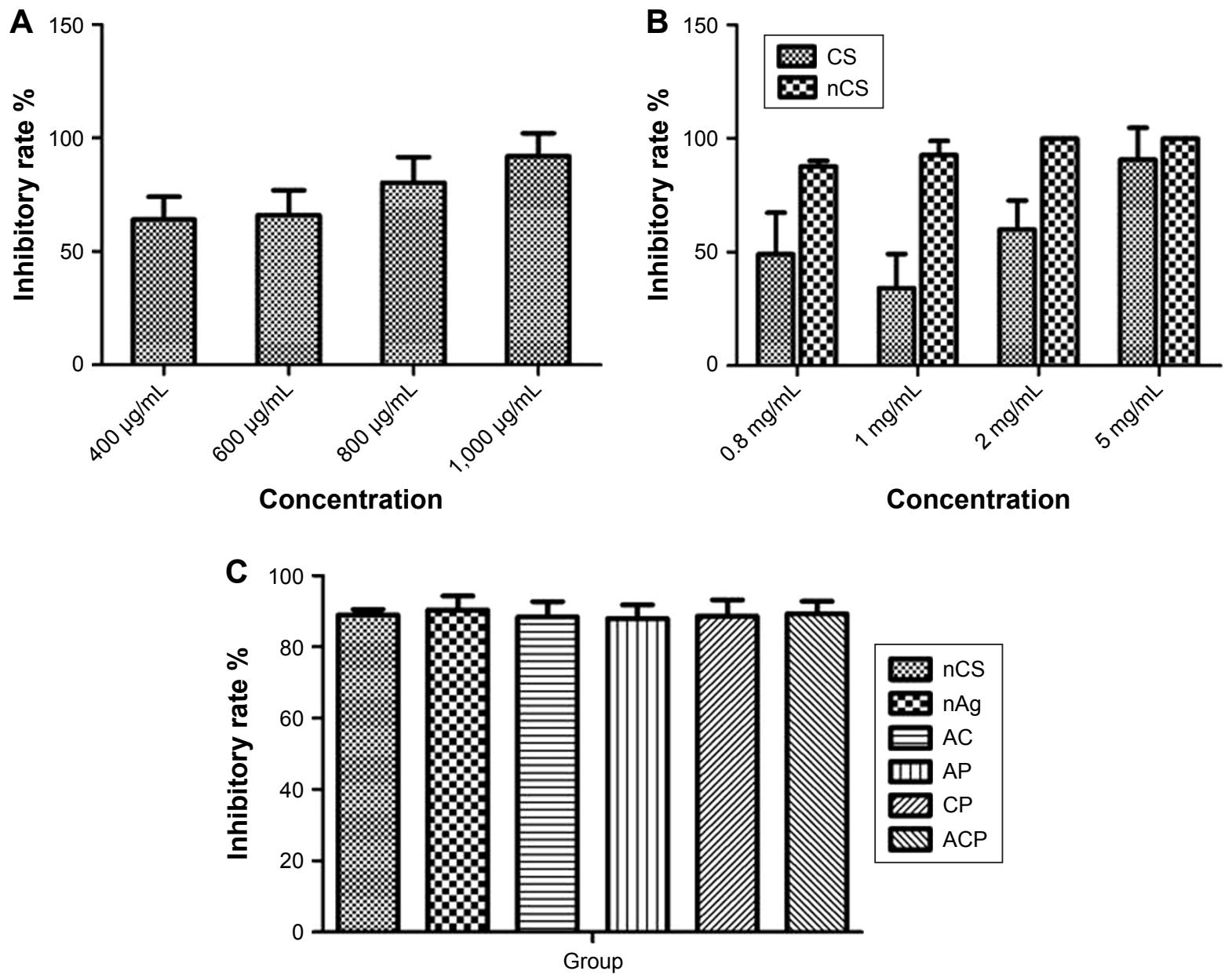

Figure 6 Antibacterial activity of different materials against Escherichia coli.

Notes: Inhibitory effect of $n A g$ against $E$. coli $(\mathbf{A})$. Inhibitory effect of $C S$ and $n C S$ against $E$. coli $(\mathbf{B})$. The influence of $n P L G A$ on the inhibitory effect of $n A g$ and $n C S$ against E. coli (C). AC (nAg+nCS); AP (nAg+nPLGA); CP (nCS+nPLGA); ACP (nAg+nCS+nPLGA).

Abbreviations: CS, chitosan; nAg, silver nanoparticles; nCS, CS nanoparticles; nPLGA, poly(lactic-co-glycolic acid) nanoparticles. 


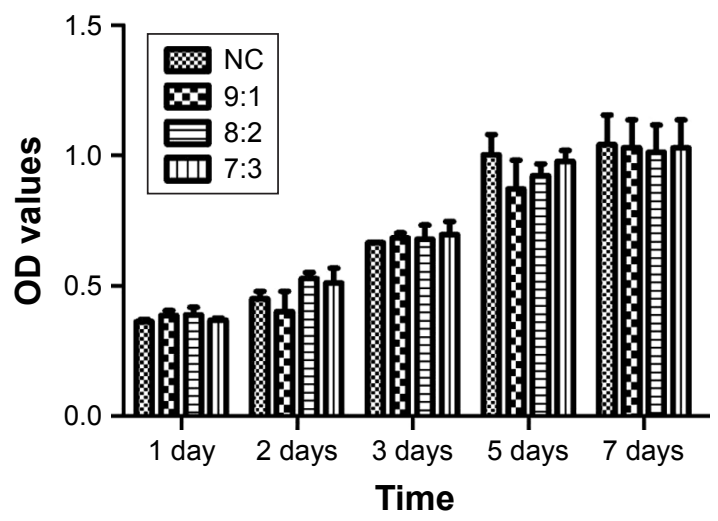

Figure 7 Effect of $n P L G A / n C S$ in different ratios on the proliferation of hPDLCs. Abbreviations: hPDLCs, human periodontal ligament cells; $\mathrm{nCS}$, chitosan nanoparticles; nPLGA, poly(lactic-co-glycolic acid) nanoparticles.

\section{H\&E staining}

At week 2, no obvious inflammatory cell formation was observed at the edge of the material and tissue. At weeks 4-8, the tissue grew into the definite area and healed without any obvious inflammation on the margin of bone defect. The position of the edge is shown by arrow in Figure 14. Bone tissue healed well at weeks 4-8. Some edges were blurred, and no inflammatory cells such as neutrophils or lymphocytes were found (Figure 14, arrow).

\section{Discussion}

The reduced alveolar bone height is difficult to restore to the ideal state, though periodontitis can be controlled by periodontal therapy. Bone tissue regeneration is important in periodontal therapy. Tissue regeneration can be induced by growth factors, ${ }^{19}$ scaffolds, ${ }^{20}$ and stem cell transplantation. ${ }^{21}$ It was found in our previous study that calcium alginate, PLGA, and CS have an excellent biocompatibility and are able to improve cell mineralization. Animal experiments have

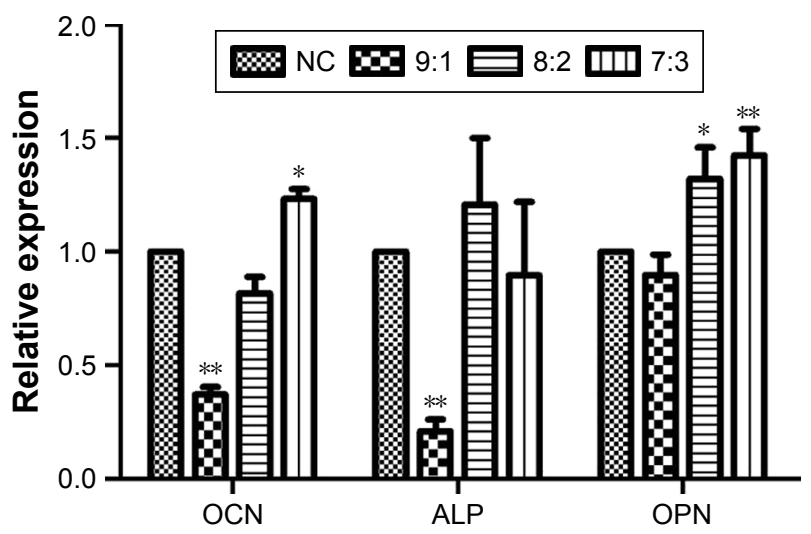

Figure 9 The relative mRNA expression of OCN, ALP, and OPN $(* P<0.05$ $* * P<0.01)$.

also shown excellent bone induction. ${ }^{22,23}$ Nanoparticles are considered as favorable analogs of human microstructure and were proven to have excellent tissue regeneration effects in the present study. Periodontitis tends to recur; however, the recurrence rate of periodontitis may be decreased through implanting drugs that can resist bacteria for a long period in the periodontal pocket. nAg are not resistant and can be released over time, and therefore, they are a good choice. In this study, PLGA, CS, and nAg were employed to investigate their effects as single components and as composites on cell proliferation and mineralization to prepare them for further experiments.

The nanoparticles of PLGA and CS were fabricated successfully, and the results of the present study suggested that some properties of nPLGA and nCS were more brilliant in comparison with PLGA and CS. The nanoparticles were nontoxic for hPDLCs similar to PLGA and CS in the first 72 hours of application. The proliferation of hPDLCs cultured with nanoparticles (nPLGA or nCS) was not different from the hPDLCs cultured with PLGA or CS. There was
NC

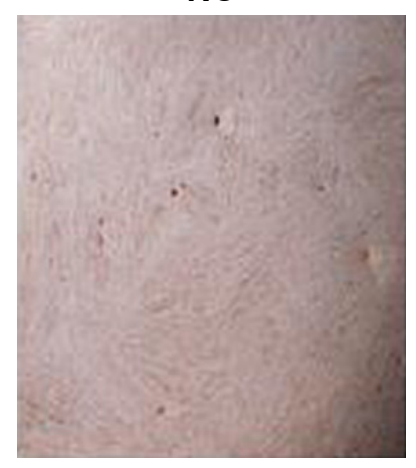

$9: 1$

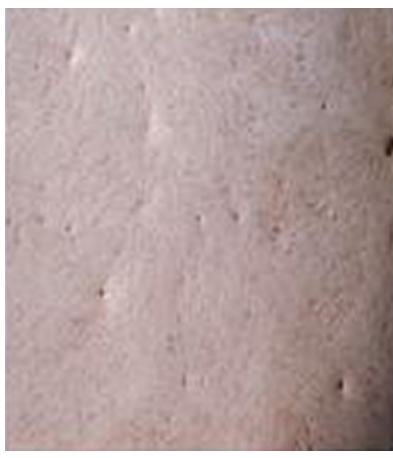

$8: 2$

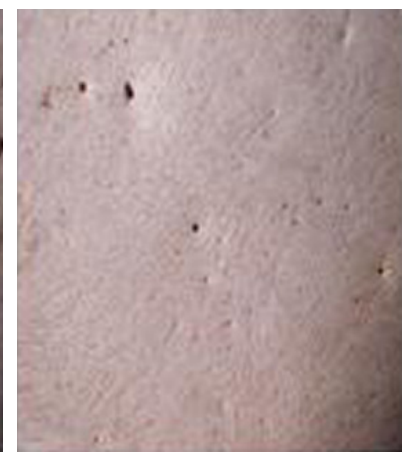

$7: 3$

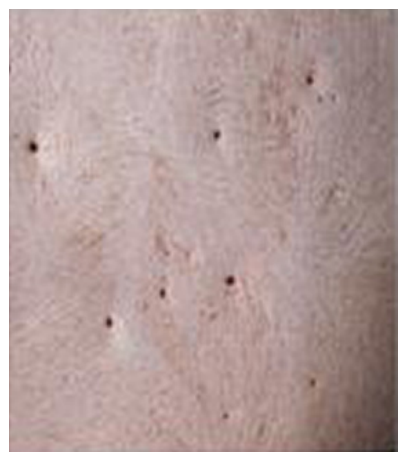

Figure 8 Alizarin Red S staining $(\times 100)$. 


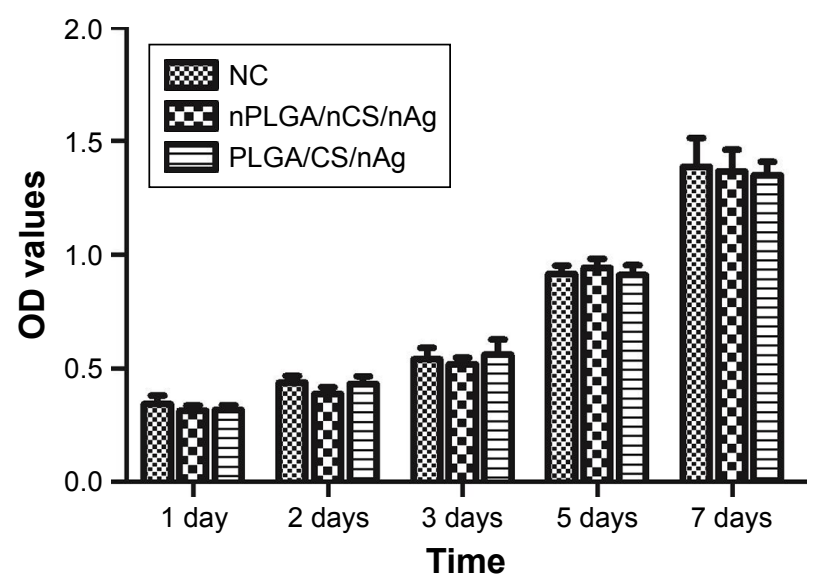

Figure 10 Effect of $n P L G A / n C S / n A g$ on the proliferation of hPDLCs compared with PLGA/CS/nAg.

Abbreviations: CS, chitosan; hPDLCs, human periodontal ligament cells; $n A g$, silver nanoparticles; nCS, CS nanoparticles; nPLGA, PLGA nanoparticles; PLGA, poly(lactic-co-glycolic acid).

no difference between the control group and nanoparticles group or between PLGA and CS group. All of these suggested that nanoparticles have excellent biocompatibility. $\mathrm{nAg}$ were also safe for hPDLCs when their concentration was not higher than $50 \mu \mathrm{g} / \mathrm{mL}$ in the study. However, $\mathrm{nAg}$ began to restrain the proliferation of hPDLCs when their concentration reached $40 \mu \mathrm{g} / \mathrm{mL}$ on the fifth day. nPLGA and $\mathrm{nCS}$ are considered as safe drug carriers. ${ }^{24-27}$ This study also confirmed that CS or PLGA had no obvious cytotoxicity on periodontal membrane cells and no impact on their proliferation and may be applied for periodontal bone tissue regeneration. The safe concentration of $\mathrm{nAg}$ was found as $40-50 \mu \mathrm{g}$.

The ARS assay showed that the red staining in all the materials groups was intense than the control group. However, analysis of the mineralization genes expression showed that the osteogenic differentiation capability of hPDLCs in the PLGA group (or CS group) was stronger than that in the nPLGA group (or nCS group). PLGA (or CS) significantly upregulated the expression of OCN and OPN, while nPLGA (or nCS) only upregulated the early expression of ALP gene. Both nanomaterials and non-nanomaterials improved the formation of cell mineralized nodules, and the degree of formation of nodules in the two groups was similar. Thus, the difference in gene expression might be found at a different point in time, or the pathway that causes cell mineralization might be different. Using this experiment, we proved that nanoparticles could be applied for regenerating periodontal tissue and forming bone tissue.

$\mathrm{nAg}$ and CS are broad-spectrum antibacterial agents with an excellent bacteriostatic activity against Grampositive and Gram-negative bacteria. nAg could express good antibacterial activity when its concentration was low. ${ }^{28,29}$ In this study, the inhibitory rate against E. coli was above $50 \%$ when the concentration of $\mathrm{nAg}$ was merely $400 \mu \mathrm{g} / \mathrm{mL}$. The antibacterial activity of nCS was much stronger than that of CS when the concentration was lower. nCS showed excellent antibacterial activity at a lower concentration. The rate of antibacterial activity of nCS and CS against E. coli was about $85 \%$ and $50 \%$, respectively, when the concentration was $0.8 \mathrm{mg} / \mathrm{mL}$. nPLGA had no influence on the antibacterial activity of $\mathrm{nAg}$ and nCS. No synergistic effect was not found in this study between $\mathrm{nAg}$ and $\mathrm{nCS}$. When used as nanoparticles, CS showed improved antibacterial effect. CS could improve the antibacterial effect in the early stage of implantation. After chitosan degradation.Nano-silver can resist bacteria for a long time. Some studies have found $\mathrm{nAg}$ to be cytotoxic. ${ }^{30,31}$ In this study, we found that the minimum inhibitory concentration of $\mathrm{nAg}$ on $E$. coli was higher than
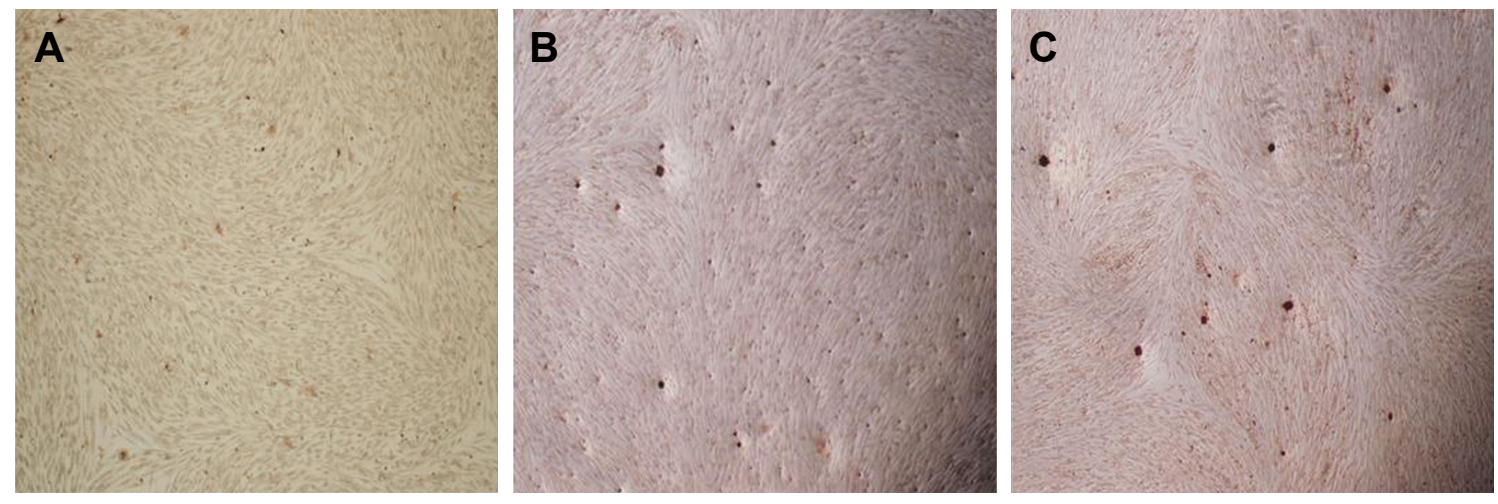

Figure I I Alizarin Red S staining $(\times 100)$.

Notes: NC (A). nPLGA/nCS/nAg (B). PLGA/CS/nAg (C).

Abbreviations: CS, chitosan; nAg, silver nanoparticles; nCS, CS nanoparticles; nPLGA, PLGA nanoparticles; PLGA, poly(lactic-co-glycolic acid). 


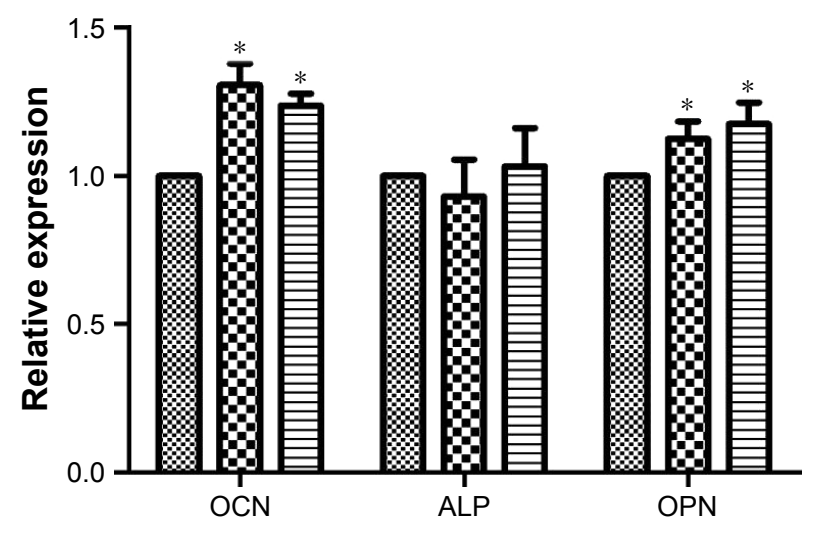

\% NC 200 nPLGA/nCS/nAg PLGA/CS/nAg

Figure 12 Real-time quantitative PCR analysis of the relative expression of osteogenic genes.

Note: $* p<0.05$.

Abbreviations: CS, chitosan; $\mathrm{nAg}$, silver nanoparticles; $\mathrm{nCS}, \mathrm{CS}$ nanoparticles; nPLGA, PLGA nanoparticles; PLGA, poly(lactic-co-glycolic acid).

the concentration that caused cytotoxicity. Unfortunately, whether this concentration causes toxicity in vivo remains to be confirmed by animal experiments.

The combination nPLGA and $\mathrm{nCS}$ in different ratios (9:1, $8: 2,7: 3)$ was also biocompatible for hPDLCs. It was nontoxic for hPDLCs and did not inhibit their proliferation. The osteogenic differentiation capability of hPDLCs cultured with this mixture increased with the increase in the concentration of nCS. In the 9:1 group, the expression of OCN and ALP was significantly downregulated, while in the 7:3 group, the expression of OCN and OPN was significantly upregulated. The combination of CS and PLGA has also been applied in some studies to improve tissue mineralization. ${ }^{32}$ However, the simultaneous preparation of PLGA and CS nanoparticles for improving the mineralization of periodontal tissues has not been reported. In this study, it was found that if these two materials are combined, the ratio of CS must be increased for better results.

In this study, $50 \mu \mathrm{g} / \mathrm{mL} \mathrm{nAg}$ mixed with the mixture of nPLGA and nCS in 7:3 ratio was cultured with hPDLCs. The mixture of the three nanomaterials showed favorable biocompatibility. It had no toxicity and no negative effect on the proliferation of hPDLCs. Both the nanoparticle mixture (nPLGA/nCS/nAg) and the non-nanoparticle mixture (PLGA/CS/nAg) upregulated the expression of bone-related genes (OCN and OPN) to some extent. The application of $\mathrm{nAg}$ in periodontal tissue regeneration has showed no obvious cytotoxicity. ${ }^{33}$ In this study, $50 \mu \mathrm{g} / \mathrm{mL}$ of $\mathrm{nAg}$ was combined
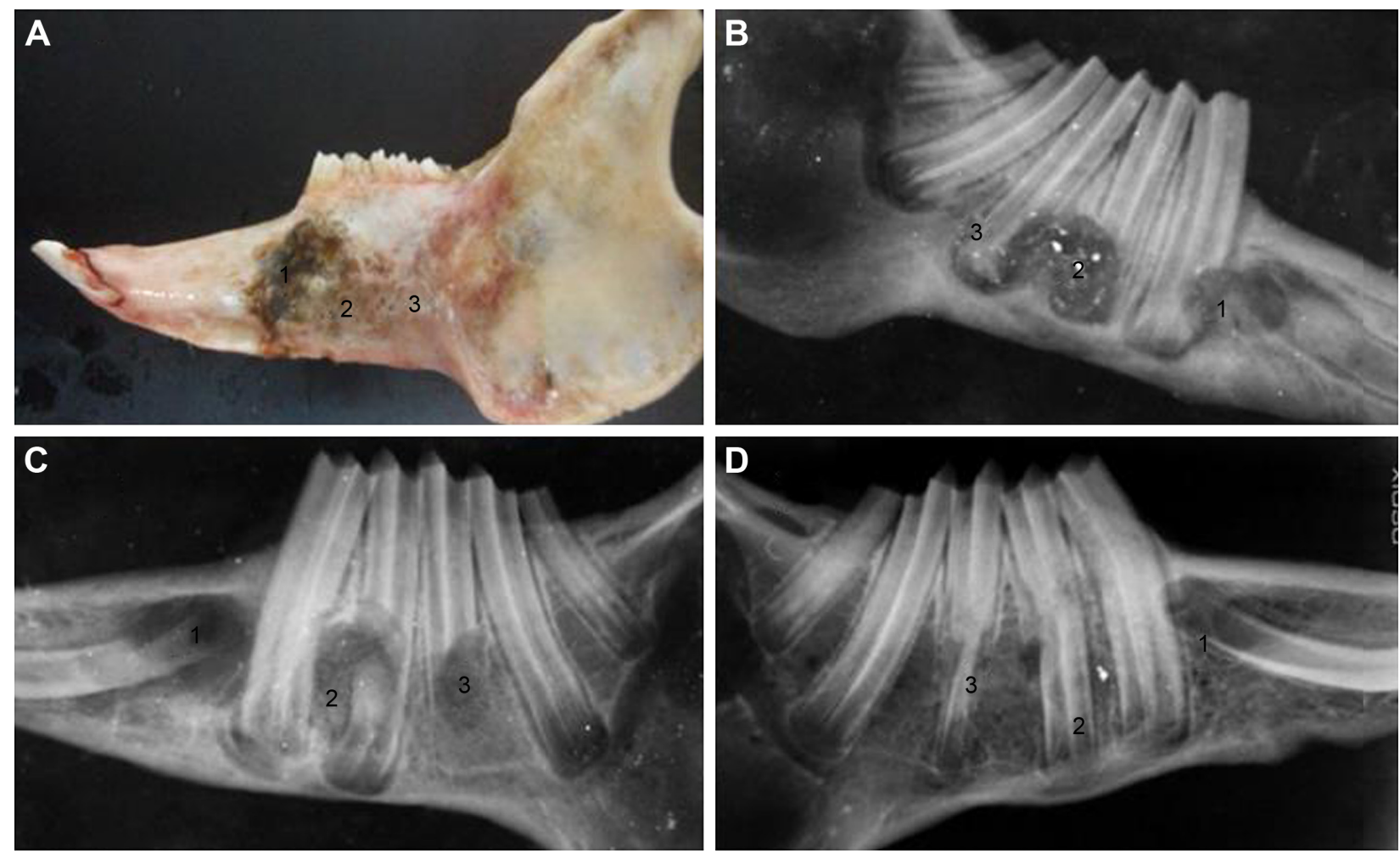

Figure 13 Analysis of bone defects.

Notes: Morphology of rabbit bone defect at 8 weeks (A). X-ray image of rabbit bone defect at 2 weeks (B). X-ray image of rabbit bone defect at 4 weeks (C). X-ray image of rabbit bone defect at 8 weeks (D). I: negative control; 2: PLGA/CS/nAg group; and 3: nPLGA/nCS/nAg.

Abbreviations: CS, chitosan; nAg, silver nanoparticles; nCS, CS nanoparticles; nPLGA, PLGA nanoparticles; PLGA, poly(lactic-co-glycolic acid). 

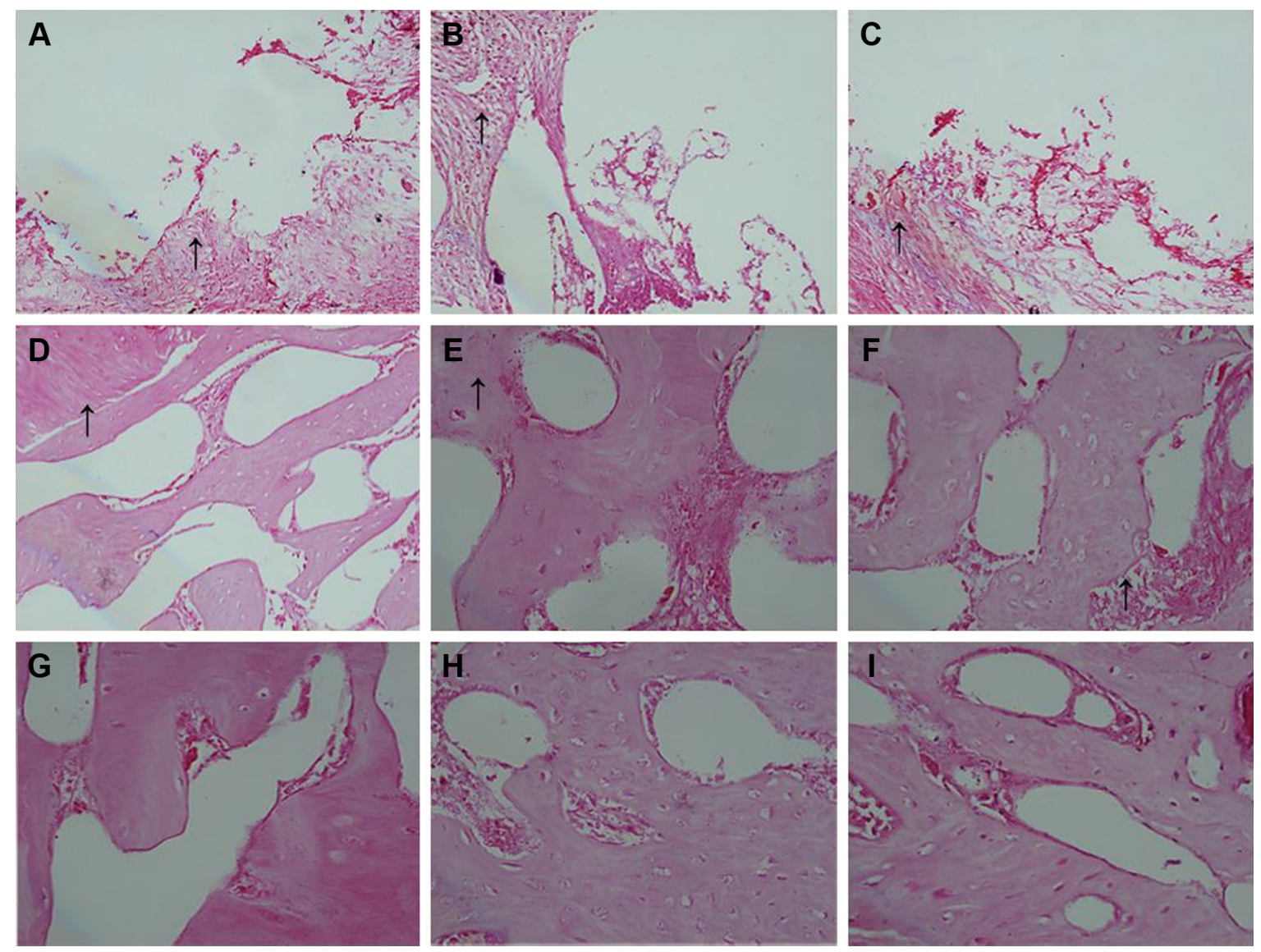

Figure 14 H\&E staining at 2, 4, and 8 weeks.

Notes: 2 weeks: negative control (A), PLGA/CS/nAg group (B), and nPLGA/nCS/nAg group (C). 4 weeks: negative control (D), PLGA/CS/nAg group (E), and nPLGA/ $\mathrm{nCS} / \mathrm{nAg}$ group $(\mathbf{F}) .8$ weeks: negative control $(\mathbf{G}), \mathrm{PLGA} / \mathrm{CS} / \mathrm{nAg}$ group $(\mathbf{H})$, and $\mathrm{nPLGA} / \mathrm{nCS} / \mathrm{nAg}$ group $(\mathbf{I})$. The arrow points to the edge of the bone defect $(\times 10)$. Abbreviations: CS, chitosan; nAg, silver nanoparticles; nCS, CS nanoparticles; nPLGA, PLGA nanoparticles; PLGA, poly(lactic-co-glycolic acid).

with $\mathrm{nPLGA} / \mathrm{nCS}$ to demonstrate that the material is not cytotoxic and contributes to cell mineralization.

After implanting the material into the mandible, we found no obvious inflammatory cell formation in the bone defect in the material group. In the X-ray film, it was found that the bone density of the experimental group was higher than that of the control group. After the rabbits were sacrificed and their mandible was removed, it was found that the healing of the mandible in the experimental group was better than that in the control group. However, in this experiment, $50 \mu \mathrm{g} / \mathrm{mL}$ of $\mathrm{nAg}$ was used and this concentration did not inhibit the growth of $E$. coli in vitro. We will further establish an animal model to verify whether the concentration of nAg in the body can change the complex bacterial flora system in the periodontal pouch. Our next experiment will verify whether the combination of three components (PLGA/CS/nAg) is superior to that of two components (PLGA/CS) in promoting cell growth of osteoblasts and inhibiting inflammatory reactions.

\section{Ethical approval, consent to participate, and publication}

Patients whose teeth were used in this study had provided written informed consent for their use, in accordance with the Declaration of Helsinki. For any patient under the age of 18, a parent or legal guardian provided consent. Ethical approval for this study was obtained from Medical Ethics Committee of Zhangshan Hospital of Xiamen University (No xmzsyyky-2018015). The submission has reported data collected from animals and humans, and all studies were conducted according to the regulations for animal experimentation issued by the State Committee of Science and Technology of the People's Republic of China.

\section{Data sharing statement}

All data generated or analyzed during this study are included in this published article. 


\section{Acknowledgment}

This study was supported by a grant from Science and Technology Planning Project of Guangdong Province (2014A020211017) and Science and Technology Planning Project of Xiamen (3502Z20174085).

\section{Author contributions}

Yanxiang Xue and Zhanchao Ye are co- first authors. All authors read and approved the final manuscript. Yanxiang Xue contributed to conception and design of the study. Xiaofang Hong contributed to data acquisition. Zhanchao Ye contributed to data analysis. Renze Shen contributed to data interpretation. Jie Gao contributed to drafting and critical revision of the article, final approval of the version to be published, and agreed to be accountable for all aspects of the work. All authors contributed to data analysis, drafting or revising the article, gave final approval of the version to be published, and agree to be accountable for all aspects of the work

\section{Disclosure}

The authors report no conflicts of interest in this work.

\section{References}

1. Mota J, Yu N, Caridade SG, et al. Chitosan/bioactive glass nanoparticle composite membranes for periodontal regeneration. Acta Biomater. 2012; 8(11):4173-4180.

2. Li Z, Ramay HR, Hauch KD, Xiao D, Zhang M. Chitosan-alginate hybrid scaffolds for bone tissue engineering. Biomaterials. 2005; 26(18):3919-3928.

3. Arpornmaeklong P, Suwatwirote N, Pripatnanont P, Oungbho K. Growth and differentiation of mouse osteoblasts on chitosan-collagen sponges. Int J Oral Maxillofac Surg. 2007;36(4):328-337.

4. Sezlev BD, Uludag H, Hasirci V. Development of PEI- RANK siRNA complex loaded PLGA nanocapsules for the treatment of osteoporosis. Tissue Eng Part A. In press 2018.

5. Wakitani S, Mitsuoka T, Nakamura N, Toritsuka Y, Nakamura Y, Horibe $\mathrm{S}$. Autologous bone marrow stromal cell transplantation for repair of full-thickness articular cartilage defects in human patellae: two case reports. Cell Transplant. 2004;13(5):595-600.

6. Casagrande S, Tiribuzi R, Cassetti E, et al. Biodegradable composite porous poly(dl-lactide-co-glycolide) scaffold supports mesenchymal stem cell differentiation and calcium phosphate deposition. Artif Cells Nanomed Biotechnol. 2017;82:1-11.

7. Mohan Raj R, Priya P, Raj V. Gentamicin-loaded ceramic-biopolymer dual layer coatings on the Ti with improved bioactive and corrosion resistance properties for orthopedic applications. J Mech Behav Biomed Mater. 2018;82:299-309.

8. Fidalgo C, Rodrigues MA, Peixoto T, Lobato JV, Santos JD, Lopes MA. Development of asymmetric resorbable membranes for guided bone and surrounding tissue regeneration. J Biomed Mater Res A. 2018; 106(8):2141-2150.

9. Shi Q, Rondon-Cavanzo EP, Dalla Picola IP, et al. In vivo therapeutic efficacy of TNF $\alpha$ silencing by folate-PEG-chitosan-DEAE/siRNA nanoparticles in arthritic mice. Int J Nanomedicine. 2018;13:387-402.
10. Ran J, Hu J, Sun G, et al. A novel chitosan-tussah silk fibroin/nanohydroxyapatite composite bone scaffold platform with tunable mechanical strength in a wide range. Int J Biol Macromol. 2016;93(Pt A): 87-97.

11. Lu Y, Li L, Zhu Y. Multifunctional copper-containing carboxymethyl chitosan/alginate scaffolds for eradicating clinical bacterial infection and promoting bone formation. ACS Appl Mater Interfaces. In press 2017.

12. Forero J, Roa E, Reyes J, Acevedo C, Osses N. Development of useful biomaterial for bone tissue engineering by incorporating nanocopper-zinc alloy (ncuzn) in chitosan/gelatin/nano-hydroxyapatite (ch/g/nhap) scaffold. Materials (Basel). 2017;10(10):1177.

13. Ramseier CA, Kinney JS, Herr AE, et al. Identification of pathogen and host-response markers correlated with periodontal disease. J Periodontol. 2009;80(3):436-446.

14. Prasad K, Lekshmi GS, Ostrikov K, et al. Synergic bactericidal effects of reduced graphene oxide and silver nanoparticles against Gram-positive and Gram-negative bacteria. Sci Rep. 2017;7(1):1591.

15. Narang J, Narang R. Nanomedicines for dental applications-scope and future perspective. Int J Pharm Investig. 2015;5(3):121-123.

16. Negri AL, Del Valle EE, Zanchetta MB, et al. Evaluation of bone microarchitecture by high-resolution peripheral quantitative computed tomography (HR-pQCT) in hemodialysis patients. Osteoporos Int. 2012; 23(10):2543-2550.

17. Ma XY, Feng YF, Wang TS. Involvement of FAK-mediated BMP-2/ Smad pathway in mediating osteoblast adhesion and differentiation on nano-HA/chitosan composite coated titanium implant under diabetic conditions. Biomater Sci. 2017;6(1):225-238.

18. Zhan X, Gao J, Liu Y, Hu J, Xue Y, Wu B. [Lactoferrin downregulates the expression of toll like receptor 4 stimulated by lipopolysaccharide in human periodontal ligament cells]. West China J Stomatol. 2014; 32(2):166-170. Chinese.

19. Jongwattanapisan P, Terajima M, Miguez PA, et al. Identification of the effector domain of biglycan that facilitates bmp-2 osteogenic function. Sci Rep. 2018;8(1):7022.

20. Maisani M, Pezzoli D, Chassande O, Mantovani D. Cellularizing hydrogel-based scaffolds to repair bone tissue: how to create a physiologically relevant micro-environment? J Tissue Eng. 2017;8: 2041731417712073

21. Mohammadrezaei D, Golzar H, Rezai RM, et al. In vitro effect of graphene structures as an osteoinductive factor in bone tissue engineering: a systematic review. J Biomed Mater Res Part A. 2018;106(8): 2284-2343.

22. Chen L, Shen R, Komasa S, et al. Drug-loadable calcium alginate hydrogel system for use in oral bone tissue repair. Int J Mol Sci. 2017; 18(5):989.

23. Shen $\mathrm{R}, \mathrm{Xu} \mathrm{W}$, Xue Y, et al. The use of chitosan/pla nano-fibers by emulsion eletrospinning for periodontal tissue engineering. Artif Cells Nanomed Biotechnol. 2018:1-12.

24. Sarangapani S, Patil A, Ngeow YK, Elsa Mohan R, Asundi A, Lang MJ. Chitosan nanoparticles' functionality as redox active drugs through cytotoxicity, radical scavenging and cellular behaviour. Integr Biol (Camb). 2018; 10(5):313-324.

25. Thandapani P, Song J, Gandin V, et al. Aven recognition of RNA G-quadruplexes regulates translation of the mixed lineage leukemia protooncogenes. ELife. 2015;4:44.

26. Arya G, Das M, Sahoo SK. Evaluation of curcumin loaded chitosan/ PEG blended PLGA nanoparticles for effective treatment of pancreatic cancer. Biomed Pharmacother. 2018;102:555-566.

27. Kussie PH, Gorina S, Marechal V, et al. Structure of the MDM2 oncoprotein bound to the p53 tumor suppressor transactivation domain. Science. 1996;274(5289):948-953.

28. Prasad K, Lekshmi GS, Ostrikov K, et al. Synergic bactericidal effects of reduced graphene oxide and silver nanoparticles against Gram-positive and Gram-negative bacteria. Sci Rep. 2017;7(1):1591. 
29. Ionescu AC, Brambilla E, Travan A, et al. Silver-polysaccharide antimicrobial nanocomposite coating for methacrylic surfaces reduces Streptococcus mutans biofilm formation in vitro. J Dent. 2015;43(12): 1483-1490.

30. Gallorini M, Di Giacomo V, Di Valerio V, et al. Cell-protection mechanism through autophagy in $\mathrm{HGFs} / \mathrm{S}$. mitis co-culture treated with Chitlac-nAg. J Mater Sci Mater Med. 2016;27(12):186.

31. Cataldi A, Gallorini M, Di Giulio M, et al. Adhesion of human gingival fibroblasts/Streptococcus mitis, co-culture on the nanocomposite system chitlac-nag. J Mater Sci Mater Med. 2016;27(5):88.
32. Liu W, Yeh YC, Lipner J, et al. Enhancing the stiffness of electrospun nanofiber scaffolds with a controlled surface coating and mineralization. Langmuir. 2011;27(15):9088-9093.

33. Srinivasan S, Kumar PT, Nair SV, Chennazhi KP, Jayakumar R. Antibacterial and bioactive alpha- and beta-chitin hydrogel/nanobioactive glass ceramic/nano silver composite scaffolds for periodontal regeneration. J Biomed Nanotechnol. 2013;9(11):1803-1816.

\section{Publish your work in this journal}

The International Journal of Nanomedicine is an international, peerreviewed journal focusing on the application of nanotechnology in diagnostics, therapeutics, and drug delivery systems throughout the biomedical field. This journal is indexed on PubMed Central, MedLine, CAS, SciSearch $®$, Current Contents ${ }^{\circledR} /$ Clinical Medicine,
Journal Citation Reports/Science Edition, EMBase, Scopus and the Elsevier Bibliographic databases. The manuscript management system is completely online and includes a very quick and fair peer-review system, which is all easy to use. Visit http://www.dovepress.com/ testimonials.php to read real quotes from published authors.

Submit your manuscript here: http://www.dovepress.com/international-journal-of-nanomedicine-journal 